\title{
Viable neutrophils release mitochondrial DNA to form neutrophil extracellular traps
}

\author{
S Yousefi ${ }^{\star 1}$, C Mihalache $^{1}$, E Kozlowski ${ }^{1}$, I Schmid $^{1}$ and HU Simon ${ }^{1}$
}

Neutrophil extracellular traps (NETs) represent extracellular structures able to bind and kill microorganisms. It is believed that they are generated by neutrophils undergoing cell death, allowing these dying or dead cells to kill microbes. We show that, following priming with granulocyte/macrophage colony-stimulating factor (GM-CSF) and subsequent short-term toll-like receptor 4 (TLR4) or complement factor 5a (C5a) receptor stimulation, viable neutrophils are able to generate NETs. Strikingly, NETs formed by living cells contain mitochondrial, but no nuclear, DNA. Pharmacological or genetic approaches to block reactive oxygen species (ROS) production suggested that NET formation is ROS dependent. Moreover, neutrophil populations stimulated with GM-CSF and C5a showed increased survival compared with resting neutrophils, which did not generate NETs. In conclusion, mitochondrial DNA release by neutrophils and NET formation do not require neutrophil death and do also not limit the lifespan of these cells.

Cell Death and Differentiation (2009) 16, 1438-1444; doi:10.1038/cdd.2009.96; published online 17 July 2009

Neutrophils have an important function in innate immune responses. They are rapidly recruited in tissues during infections, in which they kill bacteria or at least inhibit their growth. ${ }^{1}$ In recent years, it has been recognized that microbial killing does not only occur after phagocytosis, but also in the extracellular space by the formation of neutrophil extracellular traps (NETs). ${ }^{2,3}$ NET formation requires fully mature neutrophils, as immature neutrophils do not express the functional machinery required for transducing external signals. ${ }^{4}$ NETs bind and kill both Gram-positive and -negative bacteria, as well as fungi. ${ }^{5}$ NETs have been identified in appendicitis, ${ }^{2}$ sepsis, ${ }^{6}$ and pre-eclampsia, ${ }^{7}$ suggesting that these structures are of general in vivo importance.

Interestingly, similar DNA-containing extracellular structures able to bind and kill bacteria can also be generated from activated eosinophils ${ }^{8}$ and mast cells. ${ }^{9}$ Eosinophils are, like neutrophils, short-lived granulocytes. It was therefore surprising that the eosinophil extracellular structures had, at least partially, a different qualitative composition compared with NETs. For instance, the extracellular structures of eosinophils were reported to contain mitochondrial, but not nuclear, DNA, and also did not contain nuclear proteins. ${ }^{8}$ Moreover, NETs were released in association with cell membrane breaks as a consequence of neutrophil death. ${ }^{3,10}$ In contrast, the generation of the mixed extracellular DNA/granule protein structures by eosinophils did not require their cell death. ${ }^{8}$

Owing to these reported inconsistencies between neutrophils and eosinophils, we specifically characterized the nature of the released DNA during NET formation and, by a meticulous analysis, its dependence on cell death. We report that viable neutrophils release DNA on activation in a reactive oxygen species (ROS)-dependent manner, but independently of cell death. The release of DNA in this case is of mitochondrial origin.

\section{Results and discussion}

NET formation on short-term activation of mature neutrophils. Blood neutrophils were isolated and stimulated in vitro. Neutrophils were primed with granulocytemacrophage colony-stimulating factor (GM-CSF) for $20 \mathrm{~min}$ and subsequently stimulated with lipopolysaccharide (LPS) or complement factor $5 \mathrm{a}(\mathrm{C} 5 \mathrm{a})$ for $15 \mathrm{~min}$. Under these conditions, approximately $80 \%$ of the neutrophils released DNA (Figure 1a). Without GM-CSF priming or priming alone in the absence of a second stimulus, no DNA release was observed (Figure 1a). DNase resulted in the disappearance of the extracellular DNA structures (Figure 1a). As previously reported, ${ }^{2}$ we observed extracellular co-localization of DNA and the granule proteins, neutrophil elastase and myeloperoxidase (MPO), in stimulated neutrophil cultures (Figure 1b and Supplementary Video 1). In contrast, and similar to the findings earlier reported in eosinophils, ${ }^{8}$ the nuclear proteins lamin B and nuclear matrix 45 (NP-45) were not present under these conditions (Figure 1b). Moreover, poly (ADP-ribose) polymerase (PARP), another nuclear protein,

${ }^{1}$ Institute of Pharmacology, University of Bern, $\mathrm{CH}-3010$ Bern, Switzerland

*Corresponding author: S Yousefi, Institute of Pharmacology, University of Bern, Friedbühlstrasse 49, CH-3010 Bern, Switzerland.

Tel: + 4131632 3281; Fax: + 4131632 4992; E-mail: shida.yousefi@pki.unibe.ch

Keywords: apoptosis; inflammation; mitochondrial DNA; neutrophils; neutrophil extracellular traps

Abbreviations: Abs, antibodies; C5a, complement factor 5a; CGD, chronic granulomatous disease; DPI, diphenyleneiodonium; GM-CSF, granulocyte/macrophage colony-stimulating factor; LPS, lipopolysaccharide; MPO, myeloperoxidase; NET, neutrophil extracellular trap; PARP, poly(ADP-ribose) polymerase; PCR, polymerase chain reaction; PI, propidium iodide; PS, phosphatidylserine; ROS, reactive oxygen species; TLR, toll-like receptor

Received 18.5.09; revised 18.6.09; accepted 18.6.09; Edited by L Scorrano; published online 17.7.09 


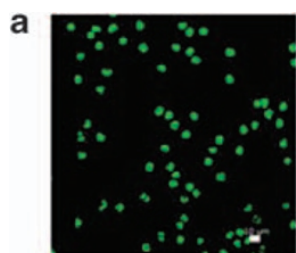

Medium

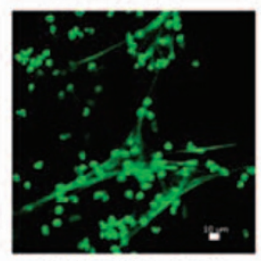

GM-CSF+LPS

b
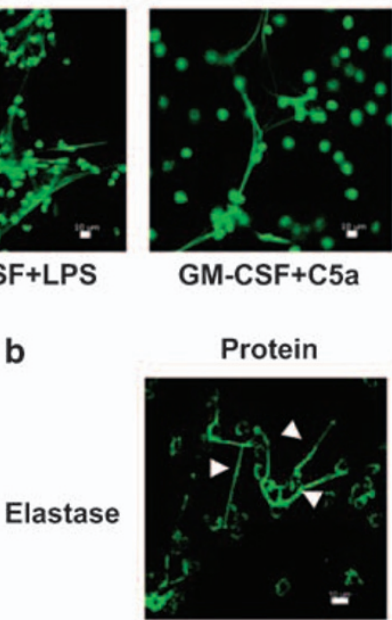

GM-CSF+C5a

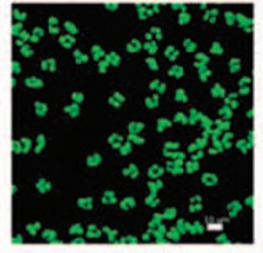

LPS

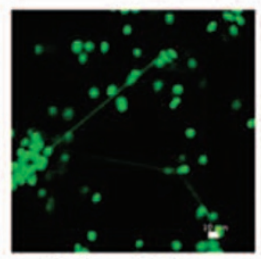

Before DNase

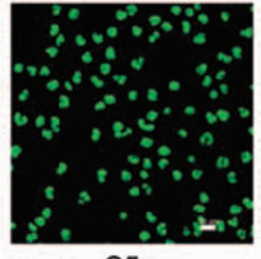

C5a

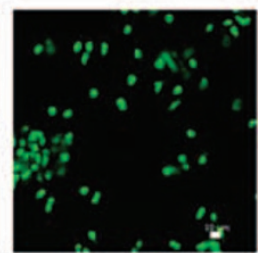

After DNase
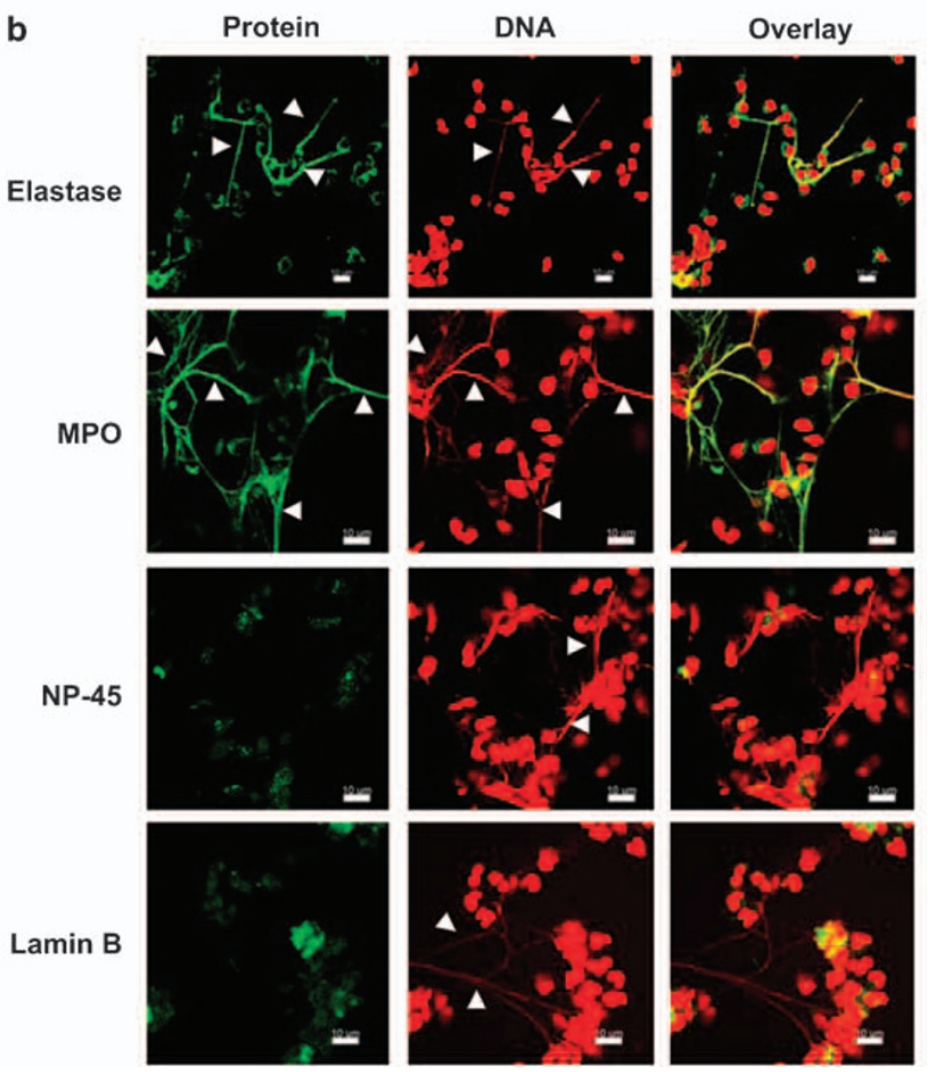

Figure 1 NETs are generated after GM-CSF priming and subsequent LPS or C5a stimulation of neutrophils (a) Purified blood neutrophils were stimulated with the indicated reagents and analyzed by confocal microscopy. Incubation with DNase resulted in disappearance of the extracellular DNA. DNA was stained by SYTO 13 in all panels. All images are projections of a z-stack. Scale bars, $10 \mu \mathrm{m}$. (b) Neutrophils were activated with GM-CSF and C5a, stained with PI and the indicated Abs, and subsequently analyzed by confocal microscopy. The extracellular deposition of DNA and the granule proteins, elastase and MPO, is indicated by arrowheads. The images are projections of a z-stack, and a z-stack movie can be seen in Supplementary Video 1 (supplementary information). Scale bars, $10 \mu \mathrm{m}$. The results shown in both panels are representative of at least three independent experiments

was also not found in these extracellular structures (data not shown). Moreover, we did not find cytoplasmic (caspase-3, $\beta$-actin), mitochondrial (cytochrome $c$, Smac), or membrane (CD15, CD16) proteins (data not shown). Taken together, NET-like extracellular structures containing DNA and granule proteins are generated following GM-CSF priming and subsequent short-term stimulation of neutrophils with LPS or $\mathrm{C5a}$.

Neutrophils release mitochondrial DNA on short-term activation. Combined two-color DNA and mitochondrial staining (SYTO 13/MitoTracker) suggested that stimulated 


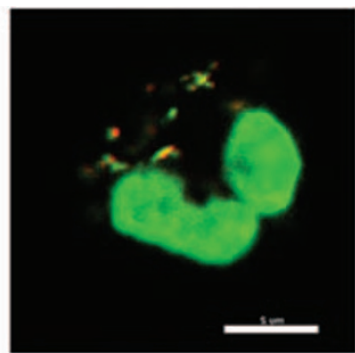

Medium

b

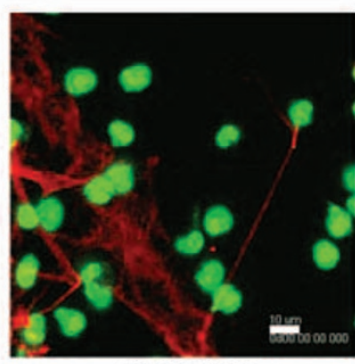

00:00.000

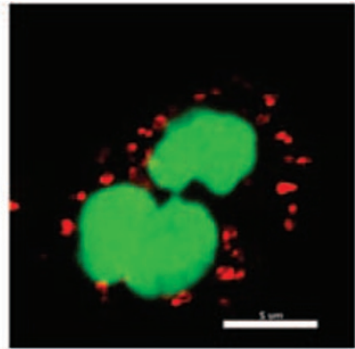

GM-CSF+LPS

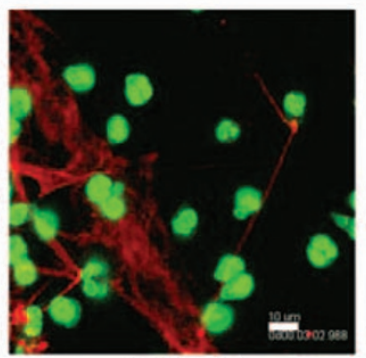

Green: SYTO 13

Red: MitoSOX Red
Green: SYTO 13

Red: MitoTracker

c
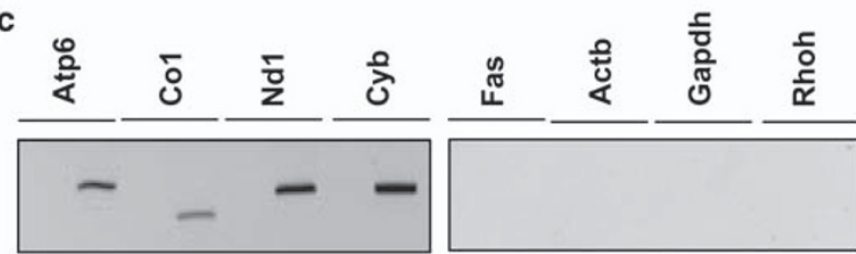

Released

DNA
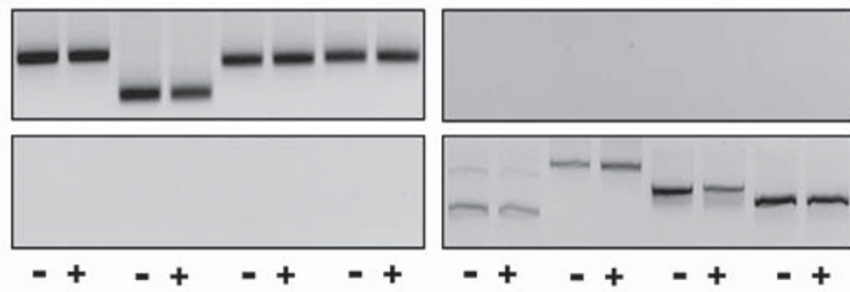

\section{Mitochondrial}

DNA

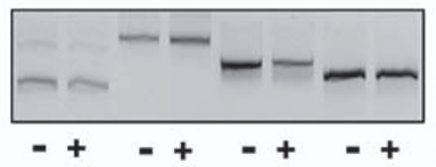

Nuclear

DNA

GM-CSF+LPS
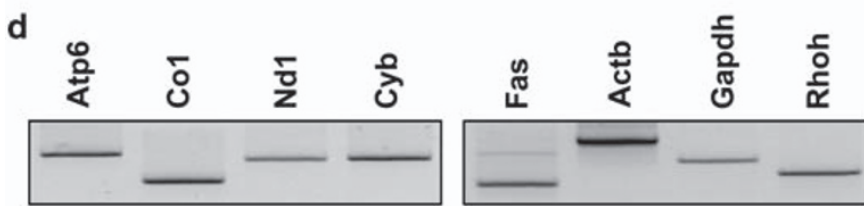

Released DNA

Figure 2 Neutrophils release mitochondrial DNA on stimulation (a) Confocal microscopy. DNA was stained with SYTO 13 and mitochondria with MitoTracker. Some DNA was identified in mitochondria (yellow). On GM-CSF/C5a stimulation of neutrophils, mitochondria are mostly shown in red. Scale bars, $10 \mu \mathrm{m}$. Images are projections of a z-stack. (b) Live-cell imaging of GM-CSF/C5a stimulated neutrophils, which were stained with SYTO 13 and MitoSOX Red before stimulation. Extracellular mitochondrial DNA is seen in red. Time elapsed is indicated. Scale bars, $10 \mu \mathrm{m}$. A complete analysis of this experiment can be seen in Supplementary Video 2 (Supplementary information). (c) PCR: Four mitochondrial (left) and four nuclear genes (right) were amplified from different DNA templates. In the released DNA, only mitochondrial genes were detectable, suggesting that activated neutrophils specifically release mitochondrial DNA. Mitochondrial and nuclear DNA isolated from purified neutrophils were used as controls. (d) PCR: In the released DNA from 36 -h neutrophil cultures (approximately $60 \%$ of the neutrophils underwent cell death), we detected both mitochondrial and nuclear DNA. The results shown in all panels are representative of at least three independent experiments

neutrophils released mitochondrial DNA (Figure 2a). Indeed, time-lapse automated confocal imaging of GM-CSF primed and C5a-stimulated neutrophils showed that perinuclear structures, most likely mitochondria, are the probable source of the released DNA from viable cells (Figure $2 \mathrm{~b}$ and Supplementary Video 2). In these experiments, we used MitoSOX Red, a fluorescent probe that enters viable cells and specifically targets mitochondria. ${ }^{11}$ The fluorescence signal of MitoSOX Red depends on superoxide production and subsequent mitochondrial DNA binding. ${ }^{11}$ MitoSOX Red particularly stained extracellular mitochondrial DNA in stimulated neutrophils and did not readily diffuse out (Figure $2 \mathrm{~b}$ and Supplementary Video 2). 
We used PCR to investigate whether the extracellular DNA contained sequences of the mitochondrial or nuclear genome. In agreement with the microscopic findings, we were able to amplify four mitochondrial but no nuclear genes from the released DNA (Figure 2c). Nuclear genes were additionally amplified from released DNA when the neutrophil population contained dead neutrophils (Figure 2d, neutrophils following 36-h culture), suggesting that selective mitochondrial DNA release is not related to cell death.

Mitochondrial DNA release by neutrophils is independent of cell death, but depends on ROS. Both the timelapse automated confocal imaging and the PCR data suggested that mitochondrial DNA release from neutrophils does not occur as a consequence of cell death. Lack of staining of activated neutrophils using a fluorescent DNA dye unable to enter viable cells (SYTOX Orange) also suggested that cell membranes after NET generation are intact (Figure 3a). Therefore, we directly investigated apoptosis and cell death, respectively, under these conditions. Within a time period of $1 \mathrm{~h}$, neither combined GM-CSF/C5a nor GM-CSF/LPS stimulation resulted in the permeabilization of the cell membrane, as all cells were propidium iodide (PI) negative (Figure $3 b$, upper panel). Both stimulation conditions also did not induce apoptosis pathways, as neutrophils did not show any evidence for phosphatidylserine (PS) redistribution (Figure 3b, upper panel), DNA fragmentation (Figure 3b, middle panel), or morphologic changes (Figure 3b, lower panel). In all these assays, we used an agonistic anti-CD95 antibody for apoptosis induction as a positive control (3-h stimulation). Moreover, we measured cell death over longer time periods in a kinetic manner. Culturing neutrophils resulted in spontaneous cell death

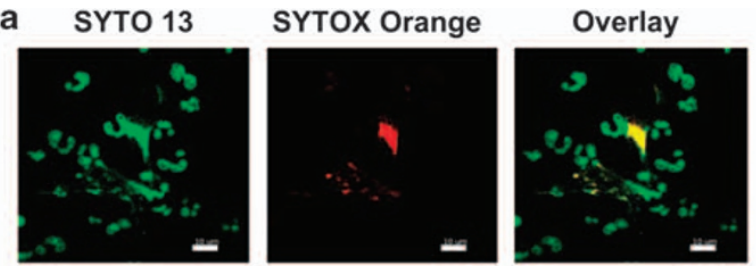

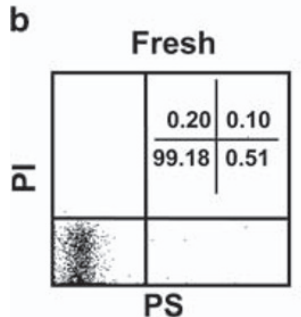

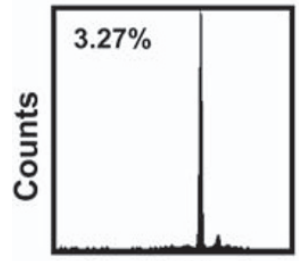

DNA

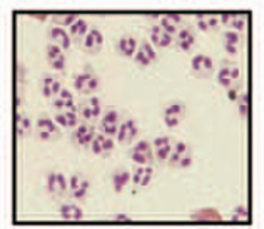

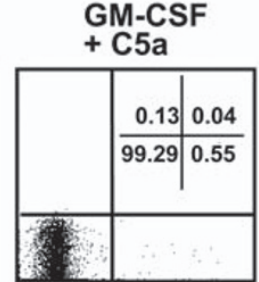

PS

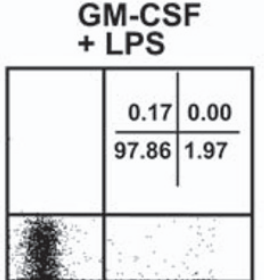

PS

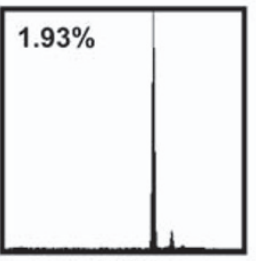

DNA
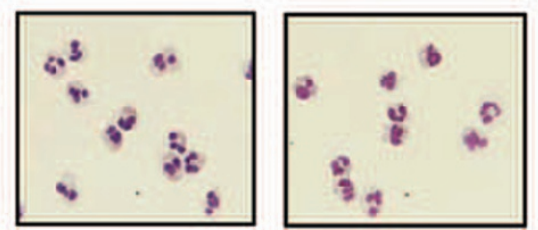
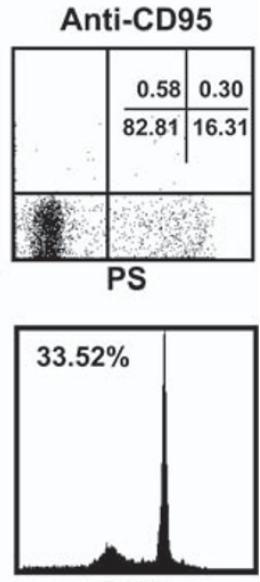

DNA

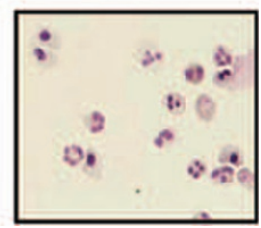

Figure 3 Release of DNA by GM-CSF/C5a or GM-CSF/LPS activated neutrophils is not associated with cell death or apoptosis (a) GM-CSF and C5a stimulated neutrophils. Cells were stained with the cell-permeable fluorescent DNA-staining dye SYTO 13 and with a fluorescent DNA dye unable to enter intact cells (SYTOX Orange). There was no evidence that SYTOX Orange would enter neutrophils in association with NET formation, indicating intact cell membranes under these conditions. Scale bars, $10 \mu \mathrm{m}$. Images are projections of a z-stack. (b) Upper panel: flow cytometric analysis of PS redistribution and cell death. Within a time period of $1 \mathrm{~h}$, both GM-CSF/C5a and GM-CSF/LPS stimulations had no effect. In contrast, 3-h stimulation of neutrophils with anti-CD95 mAb resulted in PS redistribution, indicating apoptosis, at least in a sub-population of cultured neutrophils. Middle panel: flow cytometric analysis of DNA fragmentation. Within a time period of $1 \mathrm{~h}$, both GM-CSF/C5a and GM-CSF/LPS stimulations had no effect. In contrast, 3-h stimulation of neutrophils with anti-CD95 mAb resulted in the appearance of hypodiploid DNA, most likely because of DNA fragmentation in the course of apoptosis, at least in a sub-population of cultured neutrophils. Lower panel: light microscopic analysis. No evidence for apoptosis or cell death in GM-CSF/C5a and GM-CSF/LPS stimulated neutrophils. In contrast, some of the anti-CD95 mAb-stimulated neutrophils showed evidence of apoptosis 


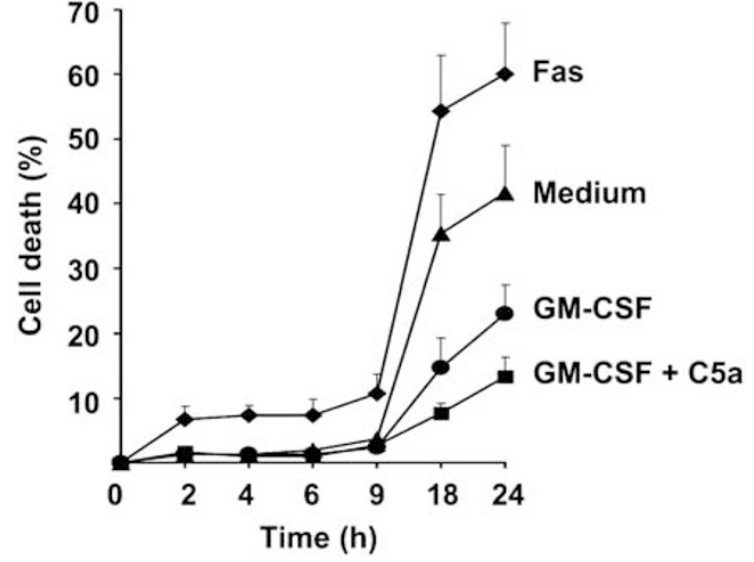

Figure 4 Stimulation of neutrophils under conditions of mitochondrial DNA release does not result in accelerated cell death. Purified blood neutrophils were cultured for the indicated times and cell death assessed by uptake of ethidium bromide and flow cytometric analysis. Values are means \pm S.D. of three independent experiments. Note that combined GM-CSF/LPS stimulation had no effect compared with medium alone (data not shown)

and GM-CSF delayed this process as earlier reported. ${ }^{12}$ Interestingly, combined GM-CSF/C5a stimulation was even more efficient that GM-CSF stimulation alone in delaying spontaneous neutrophil death (Figure 4), suggesting that mitochondrial DNA release and NET formation are not only cell death-independent processes, they also do not induce accelerated neutrophil death. Combined GM-CSF/ LPS stimulation had no effect on the viability of neutrophils compared with untreated cells (data not shown).

ROS generation is believed to be essential for NET formation following neutrophil death ${ }^{10}$ as well as the release of mitochondrial DNA by stimulated eosinophils. ${ }^{8}$ Moreover, the fluorescence signal of MitoSOX Red has been described to depend on superoxide generation and subsequent binding of the dye to mitochondrial DNA. ${ }^{11}$ We, therefore, applied diphenyleneiodonium (DPI), an inhibitor of ROS, and observed a complete block of mitochondrial DNA release from neutrophils under these conditions (Figure 5a, upper panel). We also quantitatively analyzed the released DNA in these experiments using nonpermeable SYTOX Orange, again showing that the release of DNA requires a combined GM-CSF/C5a stimulation as well as the generation of ROS (Figure 5b). Moreover, ROS-deficient neutrophils derived from patients with chronic granulomatous disease (CGD) were unable to release DNA after GM-CSF priming and subsequent $\mathrm{C} 5 \mathrm{a}$ stimulation (Figure $5 \mathrm{a}$, lower panel).

The most common physiological cell death of neutrophils is apoptosis. ${ }^{12}$ Therefore, although necrotic and necrosis-like forms of neutrophil death may also exist, ${ }^{13,14}$ it is likely that the generation of NETs under in vivo conditions is largely carried out by viable cells on appropriate activation. NET formation may also occur post mortem, but less frequent, and, perhaps, under conditions of insufficient uptake of apoptotic neutrophils. Thus, the identification of the mitochondrial DNAcontaining NETs formed by viable neutrophils constitutes an important component of innate immunity, but further study is required to understand the detailed molecular mechanisms of mitochondrial DNA release.

\section{Materials and Methods}

Reagents. Human GM-CSF was purchased from Novartis Pharma GmbH (Nürnberg, Germany). Complement factor C5a and LPS were purchased from Sigma-Aldrich (Buchs, Switzerland). DPI was purchased from CalbiochemNovabiochem Corp. (La Jolla, CA, USA). SYTOX Orange, SYTO 13, MitoSOX Red, and MitoTracker Red 580 FM probe were obtained from Invitrogen (Basel, Switzerland). DNase was purchased from Roche Diagnostics (Rotkreuz, Switzerland). Monoclonal antibodies (mAb) against elastase and lamin B were purchased from Santa Cruz Biotechnology (LabForce AG, Nunningen, Switzerland) and anti-NP-45 mAb was purchased from USBiological (Swampscott, MA, USA). Polyclonal Ab against MPO was purchased from Dako (Baar, Switzerland). Phycoerythrin (PE) and tetramethylrhodamine isothiocyanate (TRITC) - conjugated anti-mouse and anti-rabbit secondary Abs-were purchased from Jackson ImmunoResearch Laboratories (Milan Analytica, La Roche, Switzerland). Monoclonal $\mathrm{Ab}$ against $\mathrm{CD} 95$ (clone $\mathrm{CH}-11$ ) was obtained from $\mathrm{MBL}$ International Corporation (Woburn, MA, USA). The Annexin V apoptosis detection kit was purchased from BD Biosciences (Basel, Switzerland). All other reagents were, unless stated otherwise, from Sigma-Aldrich.

Neutrophil isolation. Mature blood neutrophils were isolated from peripheral blood of healthy donors and CGD patients by Ficoll-Hypaque centrifugation. ${ }^{15,16}$ Briefly, peripheral blood mononuclear cells (PBMC) were separated by centrifugation on Ficoll-Hypaque (Seromed-Fakola AG, Basel, Switzerland). The lower phase, consisting mainly of granulocytes and erythrocytes, was treated with erythrocyte lysis solution $\left(155 \mathrm{mmol} / / \mathrm{NH}_{4} \mathrm{Cl}, 10 \mathrm{mmol} / / \mathrm{KHCO}_{3}\right.$, and $0.1 \mathrm{mmol} / \mathrm{l}$ EDTA, (pH 7.3)). The resulting cell populations contained greater than $95 \%$ mature neutrophils as assessed by staining with Diff-Quik (Medion $\mathrm{GmbH}$, Düdingen, Switzerland) and light microscopy analysis.

Confocal laser-scanning microscopy. Neutrophils were seeded on 12-mm glass cover slips (BD Biosciences) and primed with $25 \mathrm{ng} / \mathrm{ml}$ GM-CSF for $20 \mathrm{~min}$. We subsequently stimulated the cells using $10^{-7} \mathrm{M}$ C5a or $0.3 \mu \mathrm{g} / \mathrm{ml}$ LPS for $15 \mathrm{~min}$. Cells were either fixed with $4 \%$ paraformaldehyde or observed in live-cell microscopy experiments. RNAs were digested by addition of $1 \mu \mathrm{g} / \mathrm{ml}$ RNase in phosphate-buffered saline for $15 \mathrm{~min}$ at room temperature. For DNA detection, we treated the slides with $5 \mu \mathrm{M}$ SYTOX Orange, $0.5 \mu \mathrm{M}$ SYTO 13 , or $5 \mu \mathrm{M}$ MitoSOX Red. For control experiments, slides were treated with 10 units $/ \mathrm{ml}$ DNase. Staining of mitochondria was carried out using $0.5 \mu \mathrm{M}$ MitoTracker probe. We washed the specimens with phosphate-buffered saline and mounted in a drop of fluorescent mounting medium (Dako). The molecules co-localizing with extracellular DNA were determined by immunofluorescence as described. ${ }^{8}$ Indirect immunostainings were carried out at $4^{\circ} \mathrm{C}$ overnight. Mouse and rabbit control antibodies, respectively, were used at the same concentrations in each experiment. Following incubation with primary $\mathrm{Ab}$, cells and tissues, respectively, were incubated with appropriate TRITC- and FITC-conjugated secondary Abs in dark at room temperature for $1 \mathrm{~h}$. The anti-fading fluorescent mounting medium (Dako) was added. Slides were covered by coverslips and analyzed by confocal laser scanning microscopy (LSM 510 and LSM 5 Exciter, Carl Zeiss Microlmaging GmbH, Jena, Germany).

Quantification of extracellular DNA. Neutrophils $\left(5 \times 10^{6}\right.$ per ml) were primed with $25 \mathrm{ng} / \mathrm{ml} \mathrm{GM}-\mathrm{CSF}$ for $20 \mathrm{~min}$ and subsequently stimulated with $10^{-7} \mathrm{M}$ C5a or $0.3 \mu \mathrm{g} / \mathrm{ml}$ LPS for $15 \mathrm{~min}$ in 96 -well plates. The medium contained $5 \mu \mathrm{M}$ SYTOX Orange. Quantitative measurements of extracellular DNA were achieved by analyzing continuous fluorescence intensity of the cultures (60-s intervals) using a Plate CHAMELEON multilabel counter.

PCR. DNA from supernatants of short-term cultured neutrophils $(25 \mathrm{ng} / \mathrm{ml}$ GM-CSF for $20 \mathrm{~min}$ plus $0.3 \mu \mathrm{g} / \mathrm{ml}$. LPS for $15 \mathrm{~min}$ or no stimulation for $40 \mathrm{~min}$ ) was purified by classical phenol, phenol/chloroform, and chloroform extraction. For control experiments, the pellets of the same cells were gently lysed and then separated into nuclear and mitochondrial fractions, ${ }^{17}$ which were both used to extract DNA after proteinase $\mathrm{K}$ treatment. Supernatants of 36 -h cultured neutrophils 


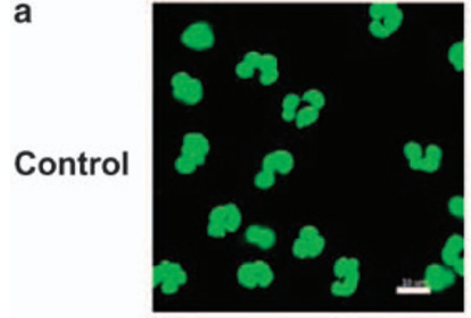

Medium

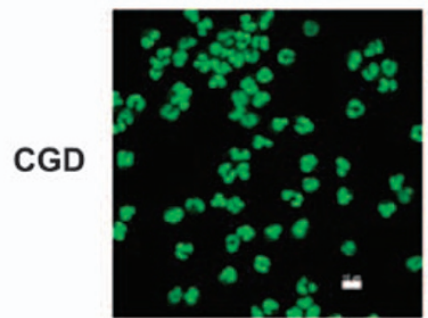

Medium

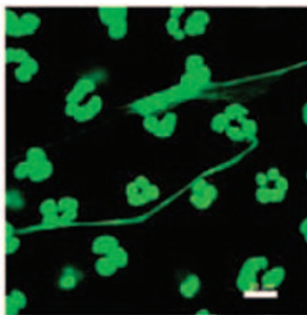

$\mathrm{GM}-\mathrm{CSF}+\mathrm{C} 5 \mathrm{a}$

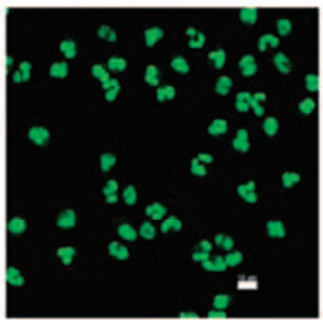

GM-CSF+C5a

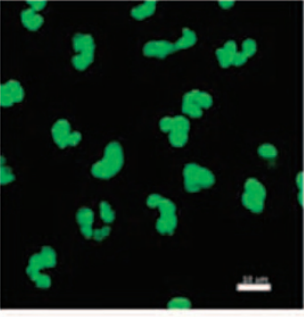

DPI

$+\mathrm{GM}-\mathrm{CSF}+\mathrm{C} 5 \mathrm{a}$

Green: SYTO 13

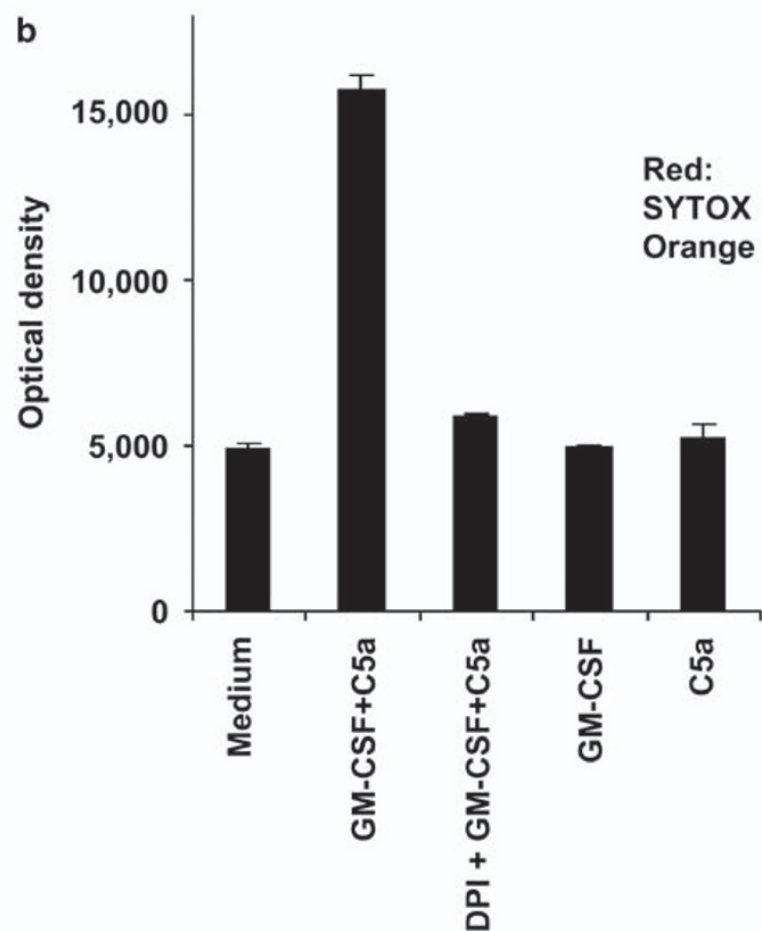

Figure 5 The release of DNA by neutrophils depends on ROS generation (a) Purified blood neutrophils were stimulated with the indicated reagents and analyzed by confocal microscopy. DPI was used to pharmacologically inhibit ROS production. In contrast to normal neutrophils, neutrophils from CGD patients were unable to release DNA on stimulation. DNA was stained by SYTO 13 in all panels. All images are projections of a z-stack. Scale bars, $10 \mu \mathrm{m}$. (b) Quantification of release DNA. Neutrophils were primed with GM-CSF and stained with SYTOX Orange. DNA was measured after 15-min stimulation with C5a using a fluorescence plate reader. Control samples without GMCSF or C5a stimulation were cultured for the same times. DPI prevented DNA release. Values are means \pm S.D. of three independent experiments

were also used for controls. The origin of the extracellular DNA was determined by amplifying four nuclear and four mitochondrial genes. Specific PCR conditions, including primers have previously been described. ${ }^{8}$

Cell death and apoptosis assays. Cell death and apoptosis assays were carried out as previously described. ${ }^{15,16}$
Acknowledgements. We greatly appreciate the help obtained from Dr. Janine Reichenbach (University Children's Hospital, Zurich, Switzerland) in blood sampling of CGD patients. This work was supported by the Swiss National Science Foundation (grant No. 310000-112078 and 310000-107526).

1. Nathan C. Neutrophils and immunity: challenges and opportunities. Nat Rev Immunol 2006; 6: 173-182. 
2. Brinkmann V, Reichard U, Goosmann C, Fauler B, Uhlemann Y, Weiss DS et al. Neutrophil extracellular traps kill bacteria. Science 2004; 303: 1532-1535.

3. Brinkmann V, Zychlinsky A. Beneficial suicide: why neutrophils die to make NETs. Nat Rev Microbiol 2007; 5: 577-582.

4. Martinelli S, Urosevic M, Daryadel A, Oberholzer PA, Baumann C, Fey MF et al. Induction of genes mediating interferon-dependent extracellular trap formation during neutrophil differentiation. J Biol Chem 2004; 279: 44123-44132.

5. Emert D, Zychlinsky A, Urban C. Fungal and bacterial killing by neutrophils. Methods $\mathrm{Mol}$ Biol 2009; 470: 293-312.

6. Clark SR, Ma AC, Tavener SA, McDonald B, Goodarzi Z, Kelly MM et al. Platelet TLR4 activates neutrophil extracellular traps to ensnare bacteria in septic blood. Nat Med 2007; 13: $463-469$.

7. Gupta AK, Hasler P, Holzgreve W, Gebhardt S, Hahn S. Induction of neutrophil extracellular DNA lattices by placental microparticles and IL-8 and their presence in preeclampsia. Hum Immunol 2005; 66: 1146-1154.

8. Yousefi S, Gold JA, Andina N, Lee JJ, Kelly AM, Kozlowski E et al. Catapult-like release of mitochondrial DNA by eosinophils contributes to antibacterial defense. Nat Med 2008; 14: 949-953.

9. von Köckritz-Blickwede M, Goldmann O, Thulin P, Heinemann K, Norrby-Teglund A, Rohde $\mathrm{M}$ et al. Phagocytosis-independent antimicrobial activity of mast cells by means of extracellular trap formation. Blood 2008; 111: 3070-3080.
10. Fuchs TA, Abed U, Goosmann C, Hurwitz R, Schulze I, Wahn V et al. Novel cell death program leads to neutrophil extracellular traps. J Cell Biol 2007; 176: 231-241.

11. Robinson KM, Janes MS, Pehar M, Monette JS, Ross MF, Hagen TM et al. Selective fluorescent imaging of superoxide in vivo using ethidium-based probes. Proc Natl Acad Sci USA 2006; 103: 15038-15043.

12. Simon HU. Neutrophil apoptosis pathways and their modification in inflammation. Immunol Rev 2003; 193: 101-110.

13. von Gunten S, Yousefi S, Seitz M, Jakob SM, Schaffner T, Seger R et al. Siglec-9 transduces apoptotic and nonapoptotic death signals into neutrophils depending on the proinflammatory cytokine environment. Blood 2005; 106: 1423-1431.

14. von Gunten S, Schaub A, Vogel M, Stadler BM, Miescher S, Simon HU. Immunologic and functional evidence for anti-Siglec-9 antibodies in intravenous immunoglobulin preparations. Blood 2006; 108: 4255-4259.

15. Conus S, Perozzo R, Reinheckel T, Peters C, Scapozza L, Yousefi S et al. Caspase-8 is activated by cathepsin $D$ initiating neutrophil apoptosis during the resolution of inflammation. J Exp Med 2008; 205: 685-698.

16. Kostylina G, Simon D, Fey MF, Yousefi S, Simon HU. Neutrophil apoptosis mediated by nicotinic acid receptors (GPR109A). Cell Death Differ 2008; 15: 134-142.

17. Yousefi S, Perozzo R, Schmid I, Ziemiecki A, Schaffner T, Scapozza L et al. Calpain-mediated cleavage of Atg5 switches autophagy to apoptosis. Nat Cell Biol 2006; 8 : $1124-1132$.

Supplementary Information accompanies the paper on Cell Death and Differentiation website (http://www.nature.com/cdd) 